\title{
Charged hadron production at LHCb
}

\author{
Óscar Boente García on behalf of the LHCb collaboration ${ }^{a, *}$ \\ ${ }^{a}$ Instituto Galego de Física de Altas Enerxías - Universidade de Santiago de Compostela, \\ Rúa de Xoaquín Díaz de Rábago s/n, Santiago de Compostela, 15782, Spain \\ E-mail: oscar.boente@usc.es
}

With a unique geometry covering the forward rapidity region, the LHCb detector provides unprecedented kinematic coverage at low $x$ for charged particles in hadron collisions. The excellent momentum resolution, vertex reconstruction, and particle identification allow precision measurements down to very low hadron transverse momentum $\left(p_{\mathrm{T}}\right)$. This contribution presents recent LHCb measurements of prompt charged hadron production in proton-proton and proton-lead collisions. Comparisons with various event generators and nPDF calculations are discussed.

*** The European Physical Society Conference on High Energy Physics (EPS-HEP2021), *** *** 26-30 July $2021 * * *$

*** Online conference, jointly organized by Universität Hamburg and the research center DESY ***

\footnotetext{
${ }^{*}$ Speaker
} 


\section{Introduction}

Charged hadrons are copiously produced in high-energy proton-proton $(p p)$ and proton-ion $(p \mathrm{~A})$ collisions at high nucleon-nucleon centre-of-mass energies $\left(\sqrt{s_{\mathrm{NN}}}\right)$. This production is mostly driven by soft-QCD processes, and quantitative predictions of the hadron production rate is challenging. Monte-Carlo event generators are generally used for those predictions, but the lack of hadron production data at forward rapidity complicates their improvement. This has an impact in experimental high-energy physics at LHC, but also in cosmic-ray physics, where these models are fundamental to study the atmospheric evolution of hadronic cascades from high-energy cosmic rays [1].

From a different perspective, the production of charged hadrons in $p \mathrm{~A}$ collisions can be studied with respect to that in $p p$ collisions. Nuclear or collective behaviour may manifest in modifications of the hadron production rate known as Cold Nuclear Matter (CNM) effects. In particular, the high hadron transverse momentum $\left(p_{\mathrm{T}}\right)$ part of the charged hadron spectra can be studied with perturbative QCD approaches [2-4]. Experimental data of the hard part of the hadron spectrum is needed to constrain these phenomenological models and to understand the origin of CNM effects.

The LHCb experiment [5, 6], located at the Large Hadron Collider (LHC) at CERN, is the only LHC detector fully instrumented between 2 and 5 units of pseudorapidity $(\eta)$. Hadron production can be studied by measuring prompt charged particles, defined as long-lived particles produced in the primary interaction or without long-lived ancestors. Long-lived charged particles, mostly $\pi, K$ and $p$, are detected using the LHCb tracking system. Raw track yields need to be corrected with reconstruction and selection efficiencies. Additionally, background contributions originating from fake tracks not corresponding to real particles and non-prompt particles are determined to correct the yields. In this contribution, two measurements of prompt charged particle production in $p p$ and proton-lead $(p \mathrm{~Pb})$ collisions at $\mathrm{LHCb}$ are reported.

\section{Prompt charged particle production in $p p$ collisions at $\sqrt{s}=13 \mathrm{TeV}$}

The production of prompt charged particles in $p p$ collisions at $\sqrt{s}=13 \mathrm{TeV}$ is measured with respect to $p_{\mathrm{T}}$ and $\eta$ [7]. Data recorded with an unbiased trigger and corresponding to an integrated luminosity of $\mathcal{L}=5.4 \mathrm{nb}^{-1}$ are used. Prompt charged particle candidates are determined from tracks reconstructed by the LHCb tracking system in the kinematic range of $2<\eta<4.8$ and $0.08<p_{\mathrm{T}}<10 \mathrm{GeV} / c$. Positively and negatively charged tracks are studied separately.

The track yield is corrected by the total efficiency and the background contributions to obtain the prompt charged particle yield. The efficiency depends on the particle type and thus on the the relative abundance of $(\pi, K, p)$ in data. These quantities are extrapolated from a previous $\mathrm{LHCb}$ measurement at different energies [8]. Background contributions are estimated with simulation samples and corrected by data-driven techniques, using background-enriched proxy samples of tracks. Fig. 1 shows the distribution of the neural-network classifier called ghost probability [9] used to construct the proxy samples for the fake track background. Similar techniques with other discriminating variables are used for the other background contributions. The uncertainty of the measurement is dominated by systematic uncertainties, ranging between $2-15 \%$ depending on the kinematic interval. The covariance matrix of their correlations is also computed. 

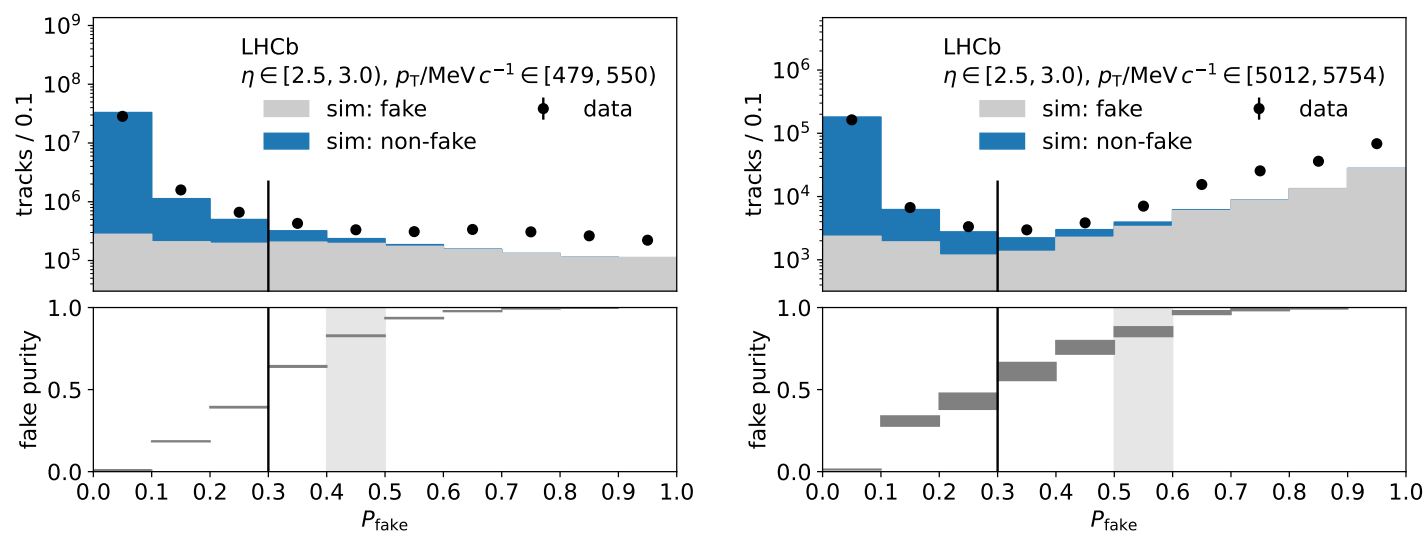

Figure 1: Distributions of ghost probability $\left(P_{\text {fake }}\right)$ in a kinematic interval at (left) low $p_{\mathrm{T}}$ and at (right) high $p_{\mathrm{T}}$. The purity of the proxy is shown in the lower subplots.
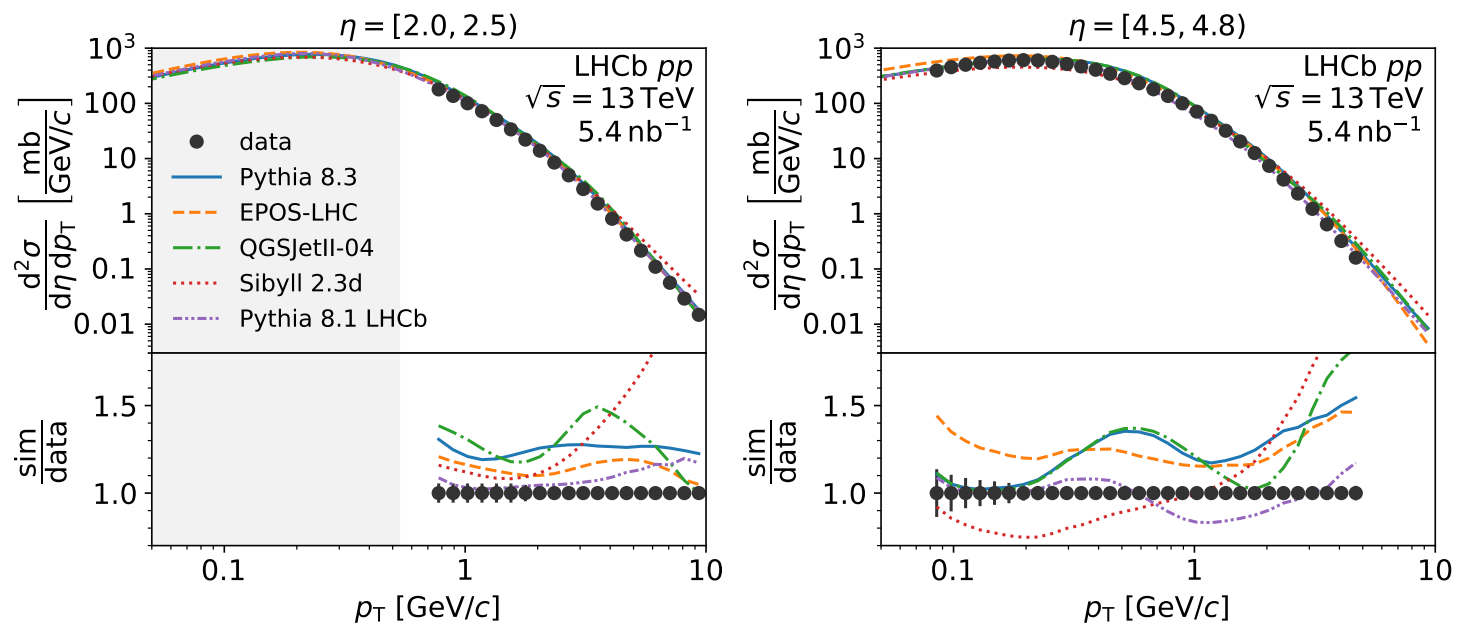

Figure 2: Differential production cross-section of prompt charge particles with respect to $p_{\mathrm{T}}$ for (left) $\eta \in[2.0,2.5]$ and (right) $\eta \in[4.5,4.8]$. The error bars indicate the total uncertainty. The ratio between model prediction and the measurement is shown in the lower subplot.

The result for double-differential cross-section is presented in Fig. 2 for two $\eta$ intervals along with the predictions from several Monte-Carlo event generators. In general, models tend to overestimate charged particle production at the LHCb acceptance. The best agreement is found with EPOS-LHC [10] and Pythia 8.1 tuned for LHCb data. The ratio of between the positively and negatively charged particle yields is best reproduced by the Pythia 8.3 model. These data provide unique constrains for future Monte-Carlo event generators.

\section{Nuclear modification factor $R_{p \mathrm{~Pb}}$ at $\sqrt{s_{\mathrm{NN}}}=5 \mathrm{TeV}$}

Prompt charged particle production is studied in $p \mathrm{~Pb}$ and $p p$ collisions at $\sqrt{s_{\mathrm{NN}}}=5 \mathrm{TeV}$ with respect to $\eta$ and $p_{\mathrm{T}}$ [11]. Double-differential production cross-sections are measured for particles 
with $p>2 \mathrm{GeV} / c, 0.2<p_{\mathrm{T}}<8.0$ and different $\eta$ coverage depending on the beam configuration: $2.0<\eta<4.8$ for $p p, 1.5<\eta<4.3$ for $p \mathrm{~Pb}$ (forward) and $-2.5<\eta<-5.2$ for $p \mathrm{~Pb}$ (backward).

Similar techniques as in [7] are used to obtain the prompt charged particle yield. A tighter selection is considered to deal with the larger background contribution from fake tracks in $p \mathrm{~Pb}$ collisions, especially in the backward configuration. Another additional difficulty is that the relative particle composition is not yet measured in $p \mathrm{~Pb}$ collisions at the LHCb acceptance. Here, the $(\pi, K, p)$ ratios are estimated with the EPOS-LHC generator [10] at the cost of an additional systematic uncertainty. The total uncertainty of the result is dominated by systematic uncertainties. For most $\left(\eta, p_{\mathrm{T}}\right)$ intervals, the total uncertainty of $p p$ and $p \mathrm{~Pb}$ production cross-sections is around $3 \%$.

The measured cross-sections allow the nuclear modification factor $R_{p \mathrm{~Pb}} \equiv \sigma_{p \mathrm{~Pb}} /\left(A \sigma_{p p}\right)$ for charged particles to be computed for the first time in the forward and backward regions at the LHC. The probed values of the parton momentum fraction $x$ in the lead nucleus range between $\left[10^{-6}, 10^{-4}\right]$ and $\left[10^{-3}, 10^{-1}\right]$ for the forward and backward region respectively. The $R_{p \mathrm{~Pb}}$ result is shown in Fig. 3 along with several phenomenological predictions. In the forward region, $R_{p} \mathrm{~Pb}$ shows a suppression of charged particle production, in general agreement with expectations from nuclear PDFs [2]. The saturation model tends to overestimate $R_{p \mathrm{~Pb}}$ in the low $p_{\mathrm{T}}$ region [3]. In the backward region, a Cronin enhancement [12] is seen for $p_{\mathrm{T}}>1.5 \mathrm{GeV} / c$. This feature is not well described by nuclear PDFs [2] or by a pQCD calculation considering parton multiple scattering [13], which reproduced the enhancement observed by the PHENIX collaboration in the backward region of proton-gold collisions [4]. The LHCb result is also compared with the ALICE result in the central $\eta$ region [14] evidencing the continuity of the $R_{p \mathrm{~Pb}}$ trend. The measurement places stringent constraints to the nuclear PDFs and saturation models over a wide $x$ range with a total relative uncertainty in the $R_{p \text { Pb }}$ determination down to $4.2 \%$.

\section{Conclusions and outlook}

The presented studies demonstrate the capabilities of the LHCb experiment to perform precise measurements of light hadron production. Both analyses provide data in kinematic regions not previously covered by other LHC experiments and are useful to constrain Monte-Carlo generators. The first measurement of the $R_{p \mathrm{~Pb}}$ in the forward and backward regions at LHC constitutes a crucial input to investigate the mechanisms behind CNM effects.

Future measurements at $\mathrm{LHCb}$ will provide additional input. The particle identification system at LHCb can cleanly separate the contribution of the different light hadrons [15], making a precise measurement of $(\pi, K, p)$ production cross-sections viable. This measurement will reduce the systematic uncertainties of the inclusive $p \mathrm{~Pb}$ measurement and also will provide additional information to Monte-Carlo generators and to study CNM effects. It is also possible to extend prompt charged particle production measurements to other collision systems accessible by $\mathrm{LHCb}$ such as ion-ion collisions. 


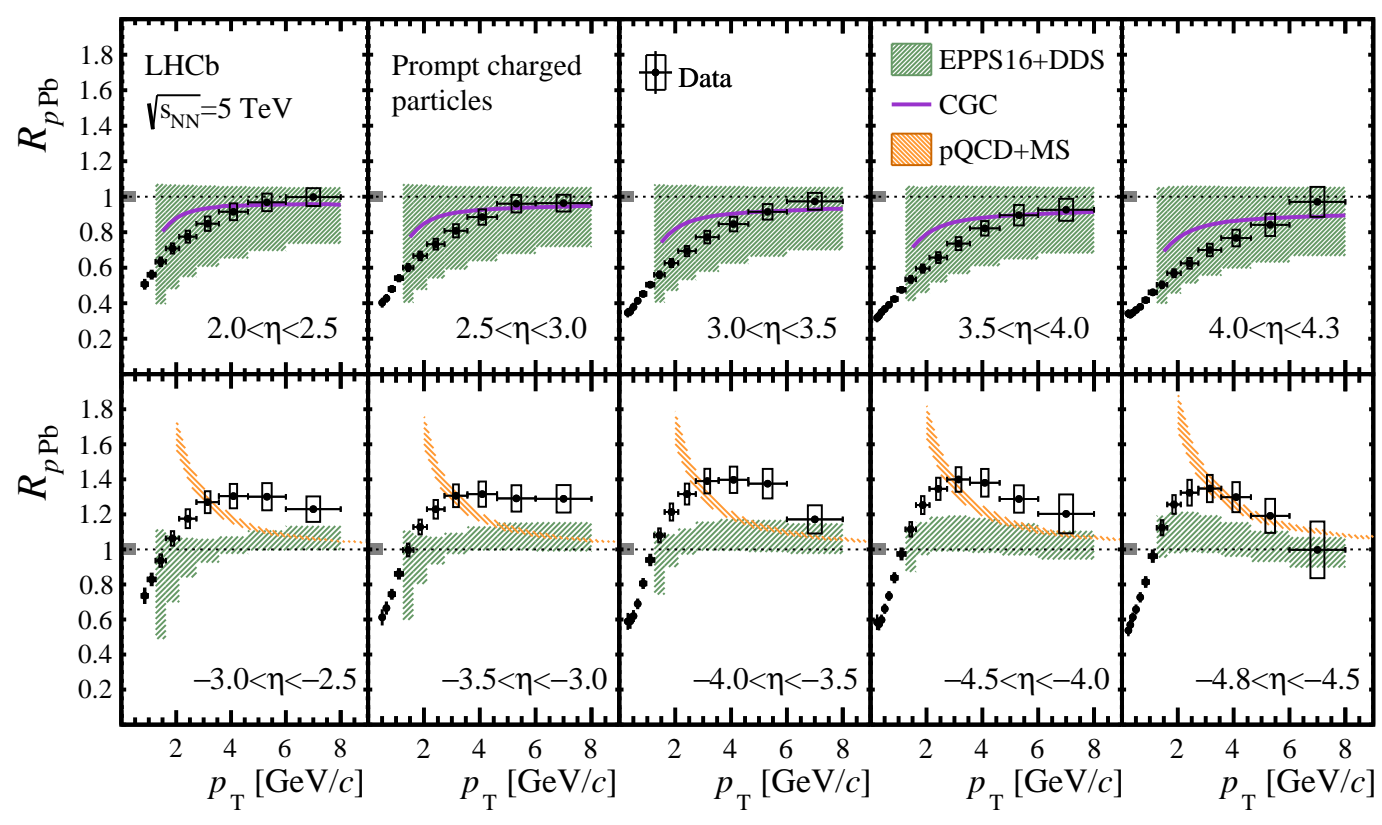

Figure 3: Nuclear modification factor as a function of $p_{\mathrm{T}}$ in different $\eta$ intervals for the (top) forward and (bottom) backward regions, compared with theoretical predictions. Vertical error bars correspond to statistical uncertainties, open boxes to uncorrelated systematic uncertainty and the filled box at $R_{p \mathrm{~Pb}}=1$ to the correlated uncertainty from the luminosity.

\section{References}

[1] J. Albrecht et al., The Muon Puzzle in cosmic-ray induced air showers and its connection to the Large Hadron Collider, 2105. 06148.

[2] I. Helenius, K. J. Eskola and H. Paukkunen, Probing the small-x nuclear gluon distributions with isolated photons at forward rapidities in p+Pb collisions at the LHC, JHEP 09 (2014) 138 [1406. 1689].

[3] T. Lappi and H. Mäntysaari, Single inclusive particle production at high energy from HERA data to proton-nucleus collisions, Phys. Rev. D88 (2013) 114020 [1309.6963].

[4] PHENIX collaboration, Nuclear-modification factor of charged hadrons at forward and backward rapidity in $p+A l$ and $p+A u$ collisions at $\sqrt{s_{N N}}=200 \mathrm{GeV}$, Phys. Rev. C101 (2020) 034910 [1906.09928].

[5] LHCв collaboration, The LHCb detector at the LHC, JINST 3 (2008) S08005.

[6] LHCв collaboration, LHCb detector performance, Int. J. Mod. Phys. A30 (2015) 1530022 LHCB-DP-2014-002, CERN-PH-EP-2014-290, [1412 . 6352]. 
[7] LHCв collaboration, Measurement of prompt charged-particle production in proton-proton collisions at $\sqrt{s}=13 \mathrm{TeV}, 2107.10090$ LHCb-PAPER-2021-010, CERN-EP-2021-110, [2107.10090].

[8] LHCв collaboration, Measurement of prompt hadron production ratios in pp collisions at $\sqrt{s}=0.9$ and $7 \mathrm{TeV}$, Eur. Phys. J. C72 (2012) 2168 LHCb-PAPER-2011-037 CERN-PH-EP-2012-171, [1206.5160].

[9] M. De Cian, S. Farry, P. Seyfert and S. Stahl, Fast neural-net based fake track rejection in the LHCb reconstruction, Tech. Rep. LHCb-PUB-2017-011, CERN, Geneva, 2017.

[10] T. Pierog, I. Karpenko, J. M. Katzy, E. Yatsenko and K. Werner, EPOS LHC: Test of collective hadronization with data measured at the CERN Large Hadron Collider, Phys. Rev. C92 (2015) 034906 [1306.0121].

[11] LHCв collaboration, Measurement of the nuclear modification factor and prompt charged particle production in $\mathrm{pPb}$ and pp collisions at $\sqrt{s_{N N}}=5 \mathrm{TeV}, 2108.13115$

LHCb-PAPER-2021-015, [2108.13115].

[12] J. W. Cronin, H. J. Frisch, M. J. Shochet, J. P. Boymond, R. Mermod, P. A. Piroue et al., Production of hadrons with large transverse momentum at 200,300, and $400 \mathrm{GeV}$, Phys. Rev. D11 (1975) 3105.

[13] Z.-B. Kang, I. Vitev, E. Wang, H. Xing and C. Zhang, Multiple scattering effects on heavy meson production in $p+A$ collisions at backward rapidity, Phys. Lett. B740 (2015) 23 [1409.2494].

[14] ALICE collaboration, Transverse momentum spectra and nuclear modification factors of charged particles in pp, $p$-Pb and Pb-Pb collisions at the LHC, JHEP 11 (2018) 013 [1802.09145].

[15] LHCв collaboration, Measurement of antiproton production in pHe collisions at $\sqrt{s_{N N}}=110$ GeV, Phys. Rev. Lett. 121 (2018) 222001 LHCb-PAPER-2018-031 CERN-EP-2018-217, [1808.06127]. 